\title{
Fractional order modelling of dynamic backlash
}

\author{
J.A. Tenreiro Machado
}

A B S T R A C T

This paper studies the dynamical properties of systems with backlash and impact phenomena. This type of non-linearity can be tackled in the perspective of the fractional calculus theory. Fractional and integer order models are compared and their influence upon the emerging dynamics is analysed. It is demonstrated that fractional models can memorize dynamical effects due to multiple micro-collisions.

Keywords:

Backlash

Impacts

Fractional calculus

Fractional derivatives

Modelling

\section{Introduction}

The phenomenon of vibration with impacts occurs in many branches of technology where it plays an useful role. However, vibrations are often undesirable, because they cause additional load and lead to faulty operation of machines. Despite the research that was carried out during the last decades, this phenomenon is not fully understood yet, due to the considerable diversity of dynamic effects involved [7,38,6,37,35,34,34,27,41, $10,28,39,18,24]$.

Fractional Calculus (FC) deals with the generalization of the differentiation and integration up to an arbitrary order $[29,33,25,31,13]$. FC is a useful tool for describing complex physical phenomena, allowing the model to take into account peculiarities that classical integer models are unable to capture $[30,9,19,44,12$, $14,26,23,3,36]$. Due to this reason, during the last decade intensive research focused the application of FC in areas such as viscoelasticity, chaos, biology, signal processing, diffusion, modelling, electromagnetism and automatic control [43,32,20,5,42,22,2,40,11,17]. In spite of the work that has been done, many areas remain to be investigated and a large volume of applications can benefit from embedding the novel concepts into refined models.

This paper investigates the dynamics of systems that contain backlash and impacts. It is shown that these non-linear phenomena can exhibit a complex dynamics and that FC is a mathematical tool well adapted for the analysis of these systems.

Bearing these ideas in mind, the present paper is organized as follows. Section 2 introduces classical methods of describing backlash. Section 3 develops a new scheme for modelling impacts by incorporating the concepts underlying FC. The results are compared with classical models of systems with static and integer-order dynamical backlash. Finally, Section 4 draws the main conclusions and addresses perspectives towards future research.

\section{Classical modelling of systems with backlash}

This section reviews the main characteristics of standard models describing systems with backlash. In Section 2.1 we start by addressing the so-called "static backlash", that involves merely geometrical effects. In Section 2.2 we recall the "dynamical backlash" that includes the phenomenon of impact.

\subsection{Static backlash}

In this sub-section we consider the phenomena of clearance without the effect of the impacts, which is usually called static backlash. The model and its input-output characteristic are shown in the right side of Fig. 1 having as input and as output the position variables $x(t)$ and $y(t)$, respectively, where $\Delta$ represents the width of the clearance and $t$ denotes time. The standard modelling of a dynamical system with backlash corresponds to a linear part, let us say for simplicity a single mass $M$ under the action of force $f(t)$ (represented in the left side of Fig. 1), followed by the static backlash [4].

Fig. 2 shows the Fourier spectrum $|\mathcal{F}\{y(t)\}|$ for a sinusoidal input force $f(t)=F \sin \left(\omega_{0} t\right)$, where $\omega_{0}$ denotes the angular frequency, and the numerical values $F=10, M=2, \omega_{0}=1$ and $\Delta=0.25$. We observe a "ordered" set of peaks located at the odd harmonics. 


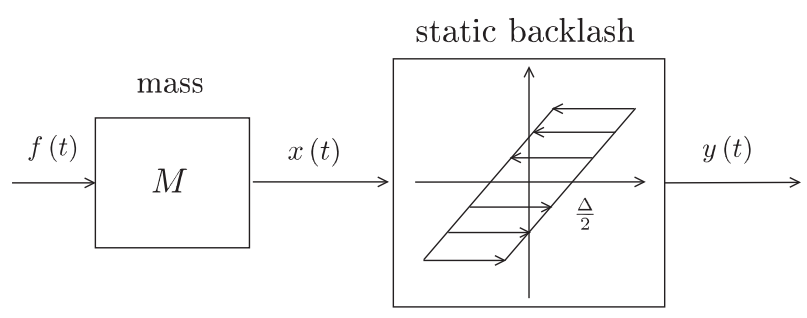

Fig. 1. Classical modelling with static backlash.

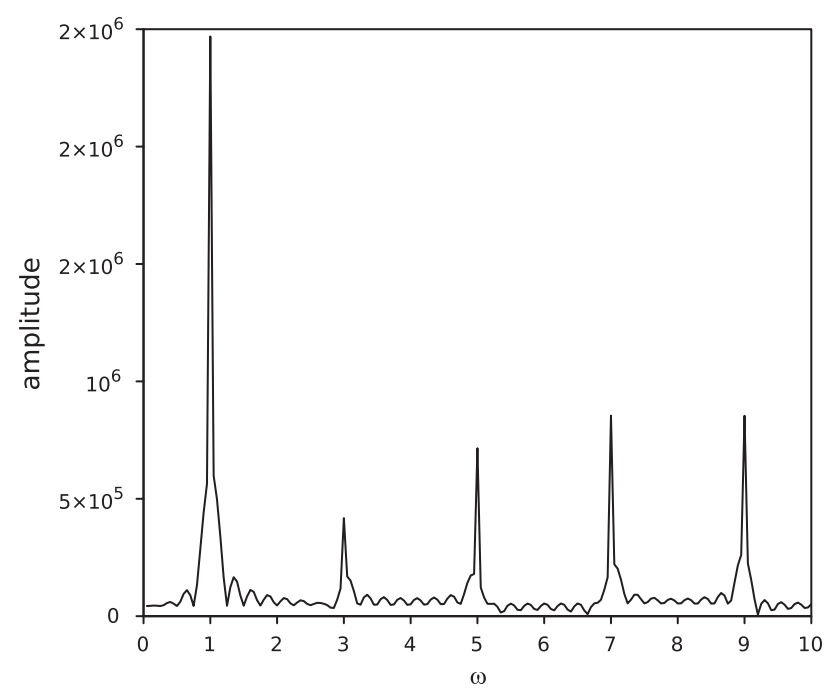

Fig. 2. Amplitude of the Fourier spectrum of the system of the static backlash for $F=10, M=2 \omega_{0}=1$ and $\Delta=0.25$.

\subsection{Dynamic backlash}

In this sub-section we consider backlash by adopting a simple description of the impact and the law of conservation of momentum $[1,21,8,15,16]$.

Let us consider two bodies colliding on surfaces that are normal to the line connecting their centres of mass, as illustrated in Fig. 3. The two bodies have velocity components only along this line and no rotational or sliding effects occur.

Fig. 4 depicts a mechanical system consisting of two masses, $M_{1}$ and $M_{2}$, subjected to backlash and impacts. Collision between the masses $M_{1}$ and $M_{2}$ occurs when $x_{1}=x_{2}-\frac{4}{2}$ or $x_{1}=x_{2}+\frac{4}{2}$. The velocities before and after the impact, $\left\{\dot{x}_{1}(t), \dot{x}_{2}(t)\right\}$ and $\left\{\dot{x}_{1}(t+\delta t), \dot{x}_{2}(t+\delta t)\right\}$, are related by means of the Newton's law:

$\dot{x}_{1}(t+\delta t)-\dot{x}_{2}(t+\delta t)=-\varepsilon\left[\dot{x}_{1}(t)-\dot{x}_{2}(t)\right]$.

where $t$ and $t+\delta t$ are two consecutive sampling instants and $\varepsilon$ is the coefficient of restitution modelling the impact phenomenon. The limit cases are the fully plastic and the ideal elastic collisions, corresponding to $\varepsilon=0$ and $\varepsilon=1$. We should note that (1) is an

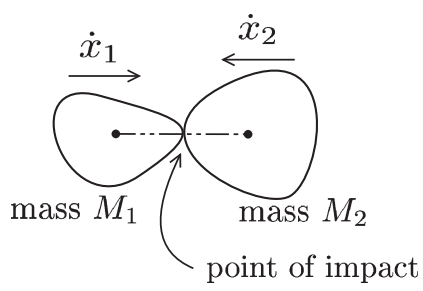

Fig. 3. Central impact of two bodies $M_{1}$ and $M_{2}$ with velocities $\dot{x}_{1}$ and $\dot{x}_{2}$.

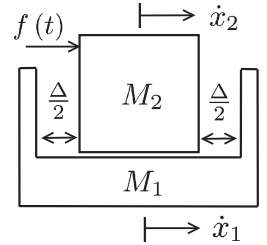

Fig. 4. System with two masses $M_{1}$ and $M_{2}$ with velocities $\dot{x}_{1}$ and $\dot{x}_{2}$ subjected to dynamic backlash with clearance $\Delta$.

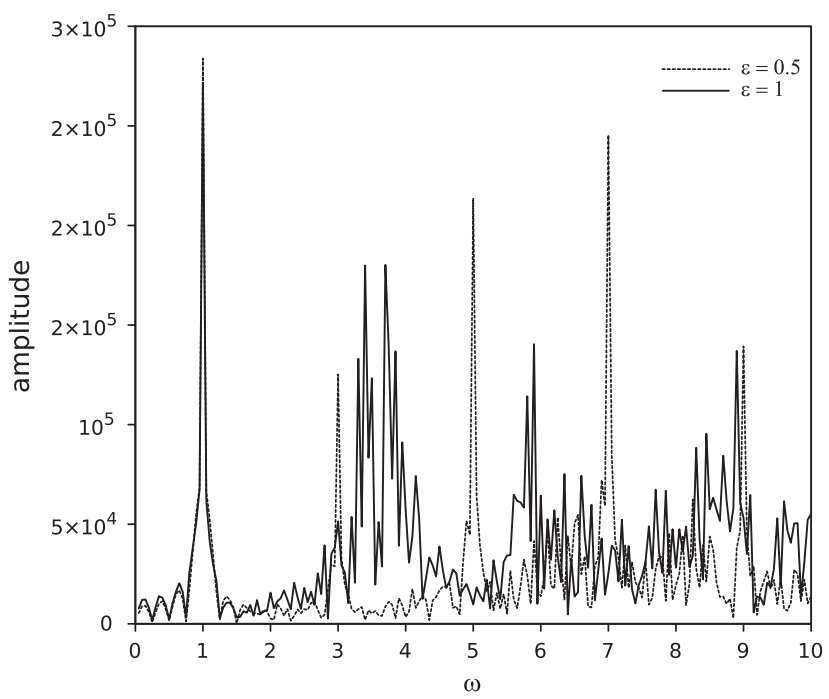

Fig. 5. Amplitude of the Fourier spectrum $\left|\mathcal{F}\left\{\ddot{x}_{2}(t)\right\}\right|$ of the dynamic backlash for $F=1, M_{1}=M_{2}=1, \omega_{0}=1, \Delta=0.25$ and $\varepsilon=\{1,0.5\}$.

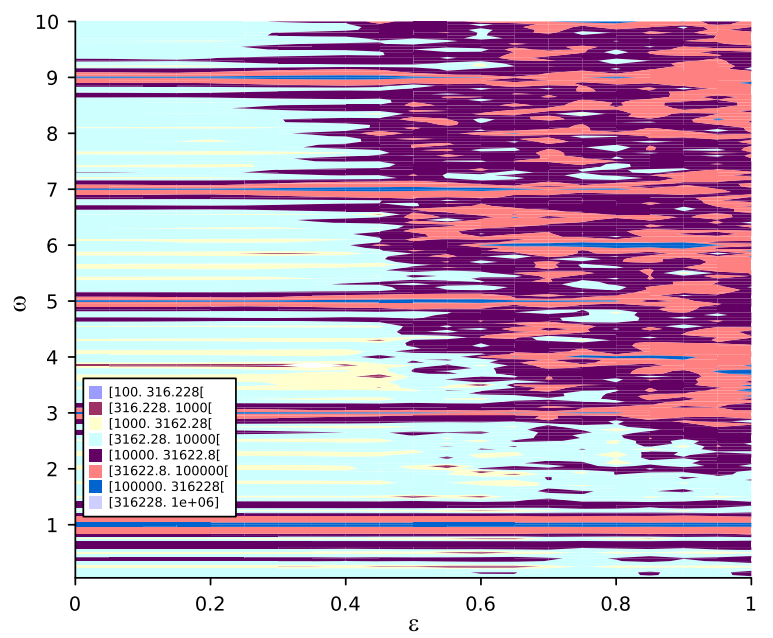

Fig. 6. Contour plot of the amplitude of the Fourier spectrum $\left|\mathcal{F}\left\{\ddot{x}_{2}(t)\right\}\right|$ versus $\{\varepsilon, \omega\}$ of the dynamic backlash for $F=1, M_{1}=M_{2}=1, \omega_{0}=1, \Delta=0.25$.

empirical description of impacts that captures in a simplified way the phenomena that occur at a microscopic level.

The principle of conservation of momentum requires that the momentum, immediately before and after the impact, is identical:

$M_{1} \dot{x}_{1}(t+\delta t)+M_{2} \dot{x}_{2}(t+\delta t)=M_{1} \dot{x}_{1}(t)+M_{2} \dot{x}_{2}(t)$.

From Eqs. (1) and (2) it yields:

$\dot{x}_{1}(t+\delta t)=\dot{x}_{1}(t) \frac{M_{1}-\varepsilon M_{2}}{M_{1}+M_{2}}+\dot{x}_{2}(t) \frac{(1+\varepsilon) M_{2}}{M_{1}+M_{2}}$,
$\dot{x}_{2}(t+\delta t)=\dot{x}_{1}(t) \frac{(1+\varepsilon) M_{1}}{M_{1}+M_{2}}+\dot{x}_{2}(t) \frac{M_{2}-\varepsilon M_{1}}{M_{1}+M_{2}}$. 


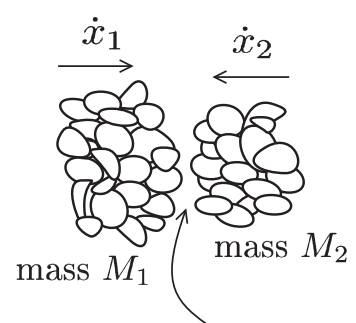

multiple points of impact

Fig. 7. Multiple impacts in the collision of two bodies with velocities $M_{1}$ and $M_{2}$ with velocities $\dot{x}_{1}$ and $\dot{x}_{2}$.

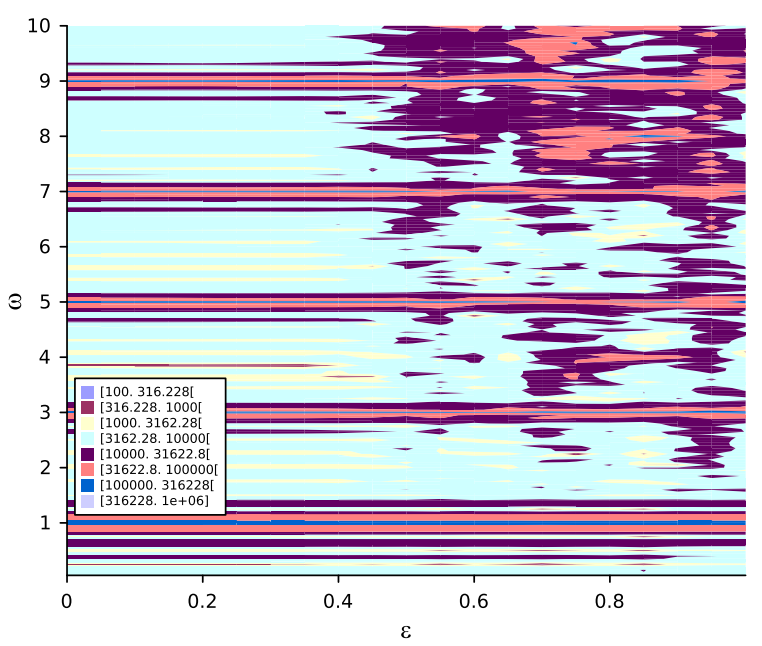

Fig. 8. Contour plot of the amplitude of the Fourier spectrum $\left|\mathcal{F}\left\{\ddot{x}_{2}(t)\right\}\right|$ versus $\{\varepsilon, \omega\}$ of the dynamic backlash for $F=1, M_{1}=M_{2}=1, \omega_{0}=1, \Delta=0.25$ and $r=2$.

Fig. 5 shows the Fourier spectrum $\left|\mathcal{F}\left\{\ddot{x}_{2}(t)\right\}\right|$ for $F=1, M_{1}=M_{2}=1$, $\omega_{0}=1, \Delta=0.25$ and $\varepsilon=\{1,0.5\}$. In the numerical simulation was adopted a total time $t_{\max }=100 \mathrm{~s}$ and a sampling period $\delta t=10^{-5} \mathrm{~s}$. We observe, in both cases, the main harmonic at $\omega=1$. However, $\varepsilon=1$ reveals a chaotic response, with the distribution of energy along all spectrum, in opposition to what occurs for $\varepsilon=0.5$.

Fig. 6 depicts the contour plot of the amplitude of the Fourier spectrum $\left|\mathcal{F}\left\{\ddot{x}_{2}(t)\right\}\right|$ versus $\{\varepsilon, \omega\}$ where we can observe the same type of behaviour.

\section{Fractional-order modelling of backlash with impact phenomena}

In this section is developed a more detailed model of impacts by including the concepts of FC.

We start by considering that the two masses $M_{1}$ and $M_{2}$ have a complex structure as represented in Fig. 7. Therefore, the central impact is constituted by a plethora of micro collisions involving the fractal structure. Furthermore, the impact is no longer instantaneous and is distributed over a small period of time. By other words, the global collision and the time elapsed during the phenomenon may be viewed as instantaneous at a macroscopic level, but are the "sum" of many small impacts that occur during a short period of time.

Our purpose is to have a model that captures easily the aforementioned phenomenon. It is well known that FC is capable of describing dynamical effects underlying fractal structures and memory relationships. We can observe that the empirical law (1) involves implicitly two consecutive time instants and a memory effect, namely the "present" velocities and the "future" velocities. Bearing these ideas in mind, expression (1) is rewritten in discrete-time:

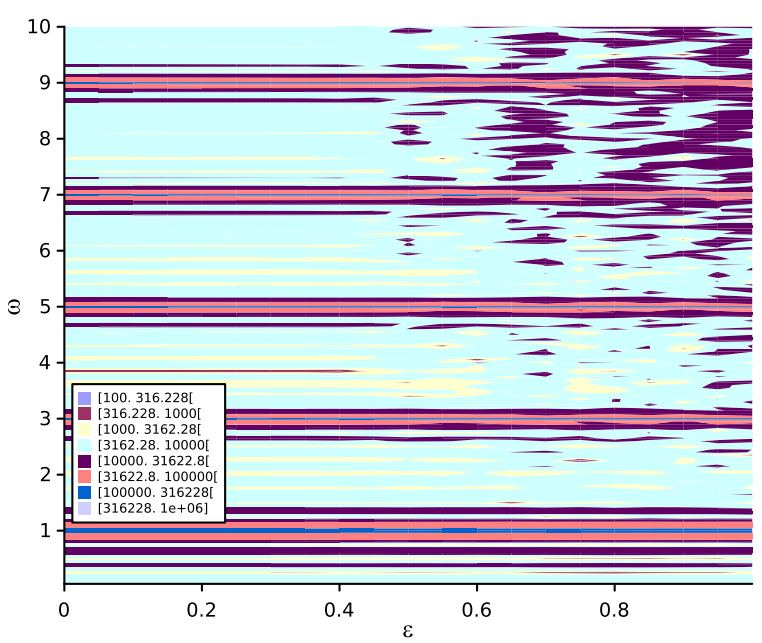

Fig. 9. Contour plot of the amplitude of the Fourier spectrum $\left|\mathcal{F}\left\{\ddot{x}_{2}(t)\right\}\right|$ versus $\{\varepsilon, \omega\}$ of the dynamic backlash for $F=1, M_{1}=M_{2}=1, \omega_{0}=1, \Delta=0.25$ and $r=10$.

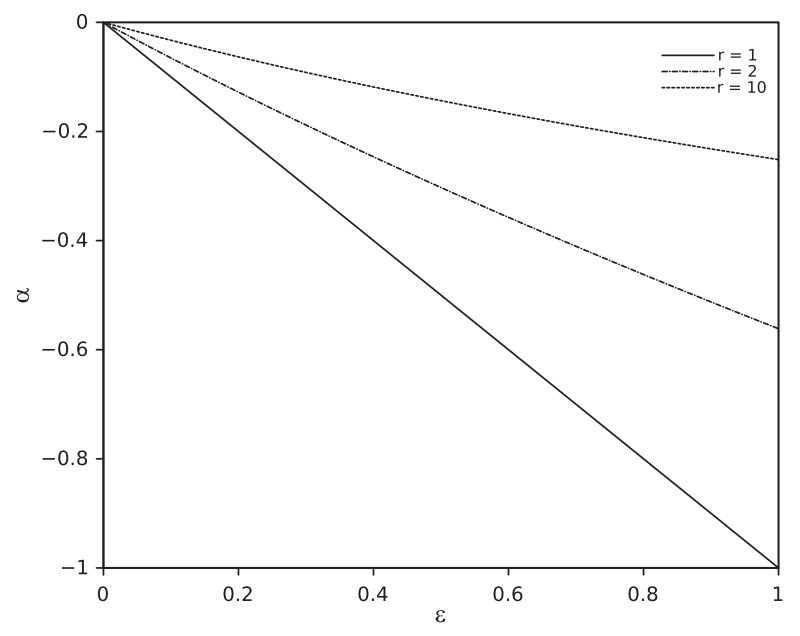

Fig. 10. Variation of $\alpha$ versus $\varepsilon$ in (9) for $r=\{1,2,10\}$.

$\dot{x}_{12}(k)=-\varepsilon \dot{x}_{12}(k-1)$.

where $\dot{x}_{12}$ denotes $\dot{x}_{1}-\dot{x}_{2}$ and $k$ and $k-1$ are two consecutive sampling instants.

This expression represents a recursive relationship between the relative velocities of the two masses before and after impact. Therefore, for representing the distributed multi-impact collision of the fractal masses represented in Fig. 5 we postulate that a more descriptive model is given by:

$\dot{x}_{12}(t+\delta t)=-\sum_{i=0}^{r} \varepsilon_{i} \dot{x}_{12}\left(t-t_{i}\right)$.

where $t_{i}$ are the time instants of impacts and $\varepsilon_{i}$ the corresponding coefficients of restitution. The values of $\varepsilon_{i}$ may be distinct for each impact, modelling the micro impacts of the two bodies during a small period of time.

The Grünwald-Letnikov definition of a fractional derivative of order $0 \leqslant \alpha \leqslant 1$ is:

$D^{\alpha} x(t)=\lim _{h \rightarrow 0} \frac{1}{h^{\alpha}} \sum_{i=0}^{\infty} \gamma(\alpha, i) x(t-i h)$

$\gamma(\alpha, i)=(-1)^{i} \frac{\Gamma(\alpha+1)}{i ! \Gamma(\alpha-i+1)}$,

where $\Gamma(\cdot)$ represents the gamma function and $h$ the time increment. In the computational implementation the time increment $h$ 


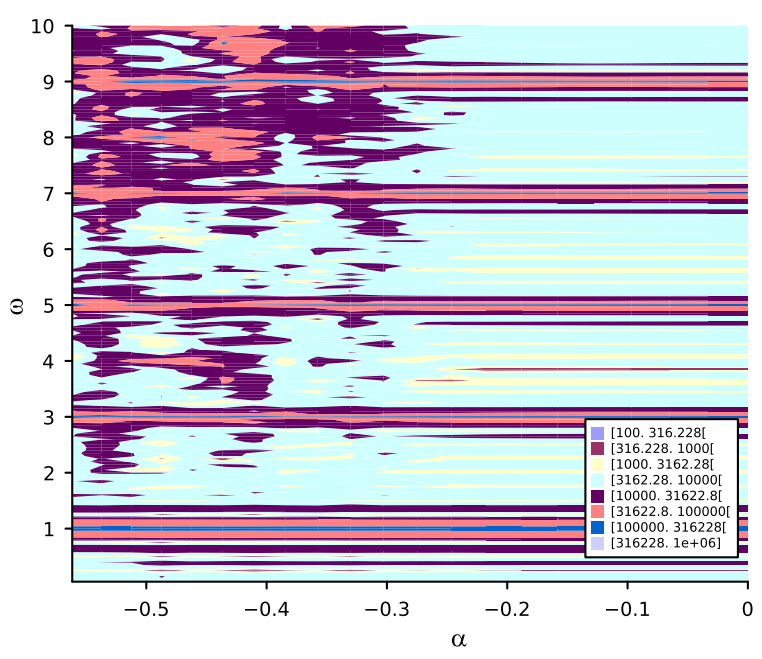

Fig. 11. Contour plot of the amplitude of the Fourier spectrum $\left|\mathcal{F}\left\{\ddot{x}_{2}(t)\right\}\right|$ versus $\{\alpha, \omega\}$ of the dynamic backlash for $F=1, M_{1}=M_{2}=1, \omega_{0}=1, \Delta=0.25$ and $r=2$.

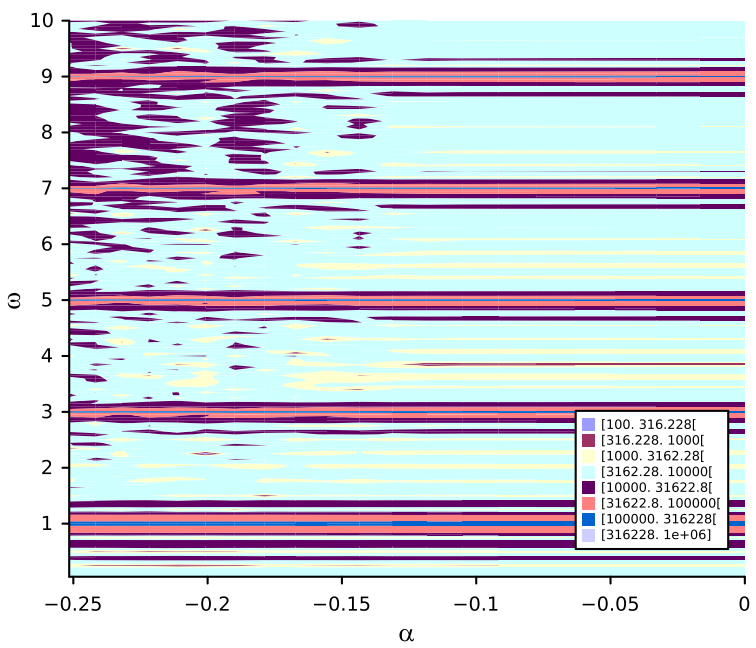

Fig. 12. Contour plot of the amplitude of the Fourier spectrum $\left|\mathcal{F}\left\{\ddot{x}_{2}(t)\right\}\right|$ versus $\{\alpha, \omega\}$ of the dynamic backlash for $F=10, M_{1}=M_{2}=1, \omega_{0}=1, \Delta=0.25$ and $r=10$.

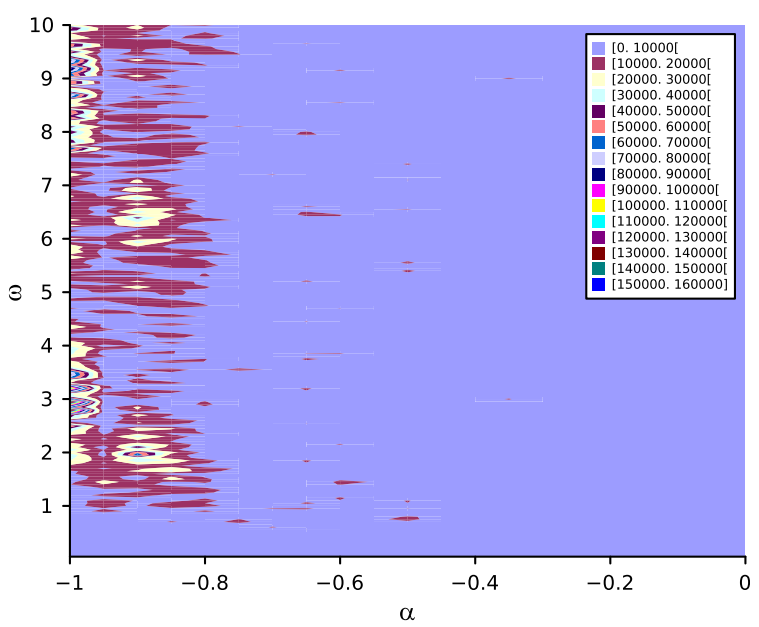

Fig. 13. Contour plot of the amplitude of the Fourier spectrum $\left|\mathcal{F}\left\{\ddot{x}_{2}(t)\right\}\right|$ versus $\{\alpha, \omega\}$ of the dynamic backlash for $F=10, M_{1}=M_{2}=1, \Delta=0.25$ and $r=1$. is approximated by the sampling period $T$ and the series is truncated as:

$D^{\alpha} x(t) \approx \frac{1}{T^{\alpha}} \sum_{i=0}^{r} \gamma(\alpha, i) x(t-i T)$,

where $r$ is the truncation order.

Inspired by approximation (8) and adopting $\gamma(\alpha, i)$ for the restitution coefficients, expression (6) can be re-written in recursive form yielding:

$\dot{x}_{12}(k)=-\sum_{i=1}^{r} \gamma(\alpha, i) \dot{x}_{12}\left(k-\tau_{i}\right)$,

where $\tau_{i}=\tau_{1}, \tau_{2}, \ldots, \tau_{r}$ denote the instants of impacts.

The system dynamics during impacts is no longer defined by the set of conditions (1) and (2). In fact, the principle of conservation of momentum (2) is still obeyed, but the impact is modelled by means of $\gamma(\alpha, i)$, that is, using the parameters $\alpha$ and $r$ that describe the fractal structure and the type of materials of the two bodies.

In order to analyse the characteristics of the fractional-order formulation we consider the experiment with parameters $F=1$, $M_{1}=M_{2}=1, \omega_{0}=1, \Delta=0.25, t_{\max }=100, \delta t=10^{-5}$ and $r=\{2,10\}$. Furthermore, for comparison with the classical dynamical backlash model it is considered $\sum_{i=0}^{r} \gamma(\alpha, i)=\varepsilon$. Figs. 8 and 9 depict the contour plots of the amplitude of the Fourier spectrum $\left|\mathcal{F}\left\{\ddot{x}_{2}(t)\right\}\right|$ versus $\{\varepsilon, \omega\}$ for $r=2$ and $r=10$, respectively. We observe, in both cases, the main harmonic located at $\omega=\omega_{0}$. The limit case $\varepsilon=1$ reveals a chaotic response, with the distribution of energy along all spectrum, in opposition with what occurs for smaller values of $\varepsilon$ that show a set of harmonics at odd frequencies. Furthermore, comparing the two figures we verify that larger values of $r$, that is, larger memories of the impact history, tend to diminish the area of chaotic behaviour.

In Figs. 8 and 9 we adjusted the parameters $\alpha$ and $r$ so that $\sum_{i=0}^{r} \gamma(\alpha, i)=\varepsilon$. Fig. 10 depicts the variation of $\alpha$ versus $\varepsilon$ in (9) for $r=\{1,2,10\}$. The representation in the $\{\varepsilon, \omega\}$ domain may not reveal clearly the effect of $\alpha$. Therefore, we redraw the contour plots in Figs. 11 and 12, representing $\left|\mathcal{F}\left\{\ddot{x}_{2}(t)\right\}\right|$ versus $\{\alpha, \omega\}$ for $r=2$ and $r=10$, respectively. We observe that the range of values of $\alpha$ varies with $r$, and that $\alpha$ plays a role similar to $\varepsilon$, while $r$ represents the amount of memory for the multiple impacts. Furthermore, larger values of $r$ lead to smaller values of $\gamma(\alpha, i)$ in (9) and to a "smoother" diminishing of the chaotic regime at the right upper of the plots.

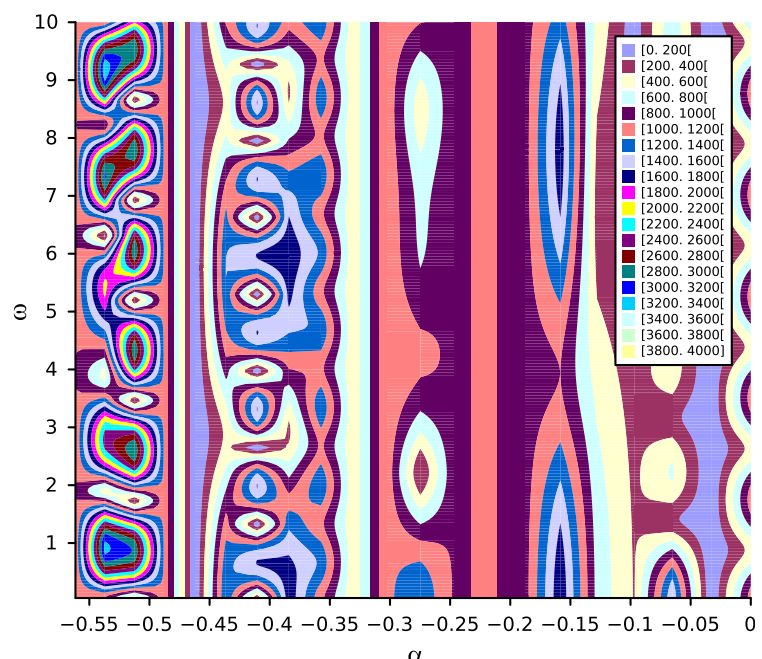

Fig. 14. Contour plot of the amplitude of the Fourier spectrum $\left|\mathcal{F}\left\{\ddot{x}_{2}(t)\right\}\right|$ versus $\{\alpha, \omega\}$ of the dynamic backlash for $F=10, M_{1}=M_{2}=1, \Delta=0.25$ and $r=2$. 


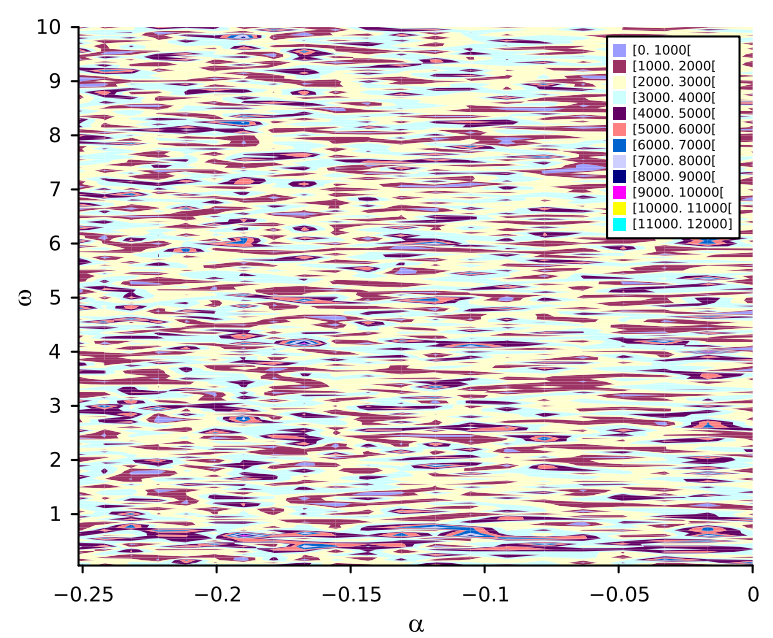

Fig. 15. Contour plot of the amplitude of the Fourier spectrum $\left|\mathcal{F}\left\{\ddot{x}_{2}(t)\right\}\right|$ versus $\{\alpha, \omega\}$ of the dynamic backlash for $F=10, M_{1}=M_{2}=1, \Delta=0.25$ and $r=10$.

We are in the presence of a non-linear system. Therefore, we have distinct responses according with the type of input signal. In this line of thought, we now test an input force $f(t)=F \cdot \operatorname{rnd}(t)$, where $\operatorname{rnd}(\cdot)$ is a function that generates white noise in the interval $[-1,+1]$. This input signal excites equally all frequency range which is visible in Figs. 13-15 representing the amplitude of the Fourier spectrum $\left|\mathcal{F}\left\{\ddot{x}_{2}(t)\right\}\right|$ versus $\{\alpha, \omega\}$ for $r=\{1,2,10\}$ and $F=10$. Comparing both cases we conclude again that the case of including a larger memory, smooths impact responses or, by other words, distributes the response along smaller components with smaller restitution coefficients $\gamma(\alpha, i)$. Therefore, while in Fig. 13 we have a strong chaotic regime at the left hand side, in Fig. 14 emerge several distributed clusters of energy and in Fig. 15 we observe a much smoother distribution along all locus.

\section{Conclusions}

This paper addressed several aspects of the phenomena involved in systems with backlash and impacts. A system with two-mass and impacts was modelled using FC concepts and the results were compared with those provided by standard models. The results revealed that fractional description can model easily more complex behaviours during impacts, namely when the surfaces under collision have a "memory" capturing the effect of multi micro impacts. These conclusions encourage further studies of non-linear systems in the perspective of FC that capture the history of the dynamic phenomena involved.

\section{References}

[1] Azenha A, Machado JT. On the describing function method and the prediction of limit cycles in nonlinear dynamical systems. J Syst Anal Modell Simul 1998;33(3):307-20.

[2] Baleanu D. About fractional quantization and fractional variational principles. Commun Nonlinear Sci Numer Simul 2009;14(6):2520-3.

[3] Baleanu D, Diethelm K, Scalas E, Trujillo JJ. Fractional calculus: models and numerical methods. Boston (Mass, USA): World Scientific; 2012.

[4] Barbosa RS, Machado JT. Describing function analysis of systems with impacts and backlash. Nonlinear Dyn 2002;29(1-4):235-50.

[5] Chen YQ, Moore KL. Discretization schemes for fractional-order differentiators and integrators. IEEE Trans Circ Syst - I: Fundam Theory Appl 2002;49(3):363-7.

[6] Choi YS, Noah ST. Periodic response of a link coupling with clearance. ASME ] Dyn Syst Measur Control 1989;111(2):253-9.

[7] Dagalakis NG, Myers DR. Adjustment of robot joint gear backlash using the robot joint test excitation technique. Int J Rob Res 1985;4(2):65-79.

[8] Duarte F, Machado JT. Describing function of two masses with backlash. Nonlinear Dyn 2009;56(4):409-13.

[9] Hilfer R. Application of fractional calculus in physics. Singapore: World Scientific; 2000.
[10] Hovland G, Hanssen S, Gallestey E, Moberg S, Brogrdh T, Gunnarsson S, Isaksson $\mathrm{M}$. Nonlinear identification of backlash in robot transmissions. In: Proc. of the 33rd int. symposium on robotics, Stockholm, Sweden; 2002.

[11] Ionescu CM. Phase constancy in a ladder model of neural dynamics. Trans Syst Man Cyber Part A - Syst Humans 2012;42(6):1543-51.

[12] Ionescu CM, De Keyser R. Relations between fractional-order model parameters and lung pathology in chronic obstructive pulmonary disease. IEEE Trans Biomed Eng 2009;56(4):978-87.

[13] Kilbas AA, Srivastava HM, Trujillo JJ. Theory and applications of fractional differential equations. North-Holland mathematics studies, vol. 204. Amsterdam: Elsevier; 2006.

[14] Li Y, Chen YQ, Podlubny I. Mittag-Leffler stability of fractional order nonlinear dynamic systems. Automatica 2009;45(8):1965-9.

[15] Lima MFM, Machado JT, Crisóstomo M. Filtering method in backlash phenomena analysis. Math Comput Modell 2009;49(4):1494-503.

[16] Lima MFM, Machado JT, Crisóstomo M. Experimental backlash study in mechanical manipulators. Robotica 2011;29(2):211-9.

[17] Luo Ying, Chen YangQuan. Fractional order motion controls. Chichester: Wiley; 2012

[18] Ma C, Hori Y. The application of fractional order control to backlash vibration suppression. In: Proc. of American control conference, Boston (Massachusetts, USA); 2004. p. 2901-6.

[19] Tenreiro Machado JA. Analysis and design of fractional-order digital control systems. Syst Anal Modell Simul 1997;27(2-3):107-22.

[20] Tenreiro Machado JA. Discrete-time fractional-order controllers. Fract Calc Appl Anal 2001;4(1):47-66.

[21] Machado JT, Galhano AMS. Fractional dynamics: a statistical perspective. ASME J Comput Nonlinear Dyn 2008;3(2):021201-1-1-5.

[22] Magin RL. Fractional calculus in bioengineering. Redding: Begell House Inc.; 2006.

[23] Mainardi F. Fractional calculus and waves in linear viscoelasticity: an introduction to mathematical models. London: Imperial College Press; 2010.

[24] Merzouki R, Davila JA, Cadiou JC, Fridman L Backlash phenomenon observation and identification. In: Proc. of American Control Conference, France; 2006. p. 3322-7.

[25] Miller KS, Ross B. An introduction to the fractional calculus and fractional differential equations. New York: John Wiley and Sons; 1993.

[26] Monje CA, Chen Y, Vinagre BM, Xue D, Feliu V. Fractional-order systems and controls: fundamentals and applications. London: Springer; 2010.

[27] M. Nordin and P. Gutman, Non-linear speed control of elastic systems with backlash. In: Proc. 39th IEEE conf. on decision and control, Sydney (Australia); 2000. p. 4060-5.

[28] Nordin M, Gutman P. Controlling mechanical systems with backlash - a survey. Automaticas 2002;38(10):1633-49.

[29] Oldham KB, Spanier J. The fractional calculus: theory and application of differentiation and integration to arbitrary order. New York: Academic Press; 1974.

[30] Oustaloup A. La commande CRONE: commande robuste d'ordre non entier. Hermès (Paris, France); 1991.

[31] Podlubny I. Fractional differential equations. An introduction to fractional derivatives, fractional differential equations, to methods of their solution, mathematics in science and engineering, vol. 198. San Diego: Academic Press; 1998.

[32] Podlubny I. Fractional-order systems and $\mathrm{PI}^{\lambda} \mathrm{D}^{\mu}$-controllers. IEEE Trans Autom Control 1999;44(1):208-13.

[33] Samko SG, Kilbas AA, Marichev OI. Fractional integrals and derivatives: theory and applications. Amsterdam: Gordon and Breach Science Publishers; 1993.

[34] Sarkar N, Ellis RE, Moore TN. Backlash detection in geared mechanisms: modeling, simulation, and experimentation. Mech Syst Signal Process 1997;11(3):391-408.

[35] Seidl DR, Lam S, Putman JA, Lorenz RD. Neural network compensation of gear backlash hysteresis in position-controlled mechanisms. IEEE Trans Ind Appl 1995;31(6):1475-83

[36] Sheng H, Chen Y, Qiu T. Fractional processes and fractional-order signal processing: techniques and applications. London: Springer; 2012.

[37] Stein JL, Wang C. Automatic detection of clearance in mechanical systems: theory and simulation. In: Proc. American control conference, Seattle (Washington, USA); 1995. p. 1737-45.

[38] Stepanenko Y, Sankar TS. Vibro-impact analysis of control systems with mechanical clearance and its application to robotic actuators. ASME J Dyn Syst Measur Control 1986;108(1):9-16.

[39] Su C, Oya M, Hong H. Stable adaptive fuzzy control of nonlinear systems preceded by unknown backlash-like hysteresis. IEEE Trans Fuzzy Syst 2003;11(1):1-8.

[40] Tarasov VE. Fractional dynamics: applications of fractional calculus to dynamics of particles, fields and media. New York: Springer; 2010.

[41] Trendafilova I, Van Brussel H. Non-linear dynamics tools for the motion analysis and condition monitoring of robot joints. Mech Syst Signal Process 2001;15(6):1141-64.

[42] West B, Bologna M, Grigolini P. Physics of fractal operators. New York: Springer; 2003

[43] Westerlund S, Ekstam L. Capacitor theory. IEEE Trans Dielectrics Electr Insulat 1994;1(5):826-39.

[44] Zaslavsky GM. Hamiltonian chaos and fractional dynamics. Oxford: Oxford University Press; 2005. 